\title{
The Research on Psychological Space of Library Based on Visual Perception
}

\author{
Y. D. Ding \\ Library, Yanshan University, Qinhuangdao, Hebei, China
}

\begin{abstract}
Space as a Service has become the consensus of modern library, how to plan the library reading space to make it fit the spatial form of reader psychology desired, become the key point to build the campus "The Third Space". The article investigated the psychological needs of the reader to reading space based humanism, combined with development trend of reading space in recent years, discusses the necessity to create a psychological space in physical space of the library. By introducing the concept of Gestalt Psychology and Visual Perception, discussed how to use similarity, closure, gestalt, proximity four principles to create a psychological space, meet readers basic needs while taking into account the multi-level, individual needs.
\end{abstract}

Keywords - library, reading space, psychological space, visual perception, gestalt psychology

\section{基于视知觉的图书馆心理空间研究}

\author{
丁玉东 \\ 燕山大学图书馆, 秦皇岛, 河北, 中国
}

摘 要 空间作为服务已成为现代图书馆建设的共识, 如何规划图书馆的阅览空间使之契合读者心理期望的空间形态, 成为打造校 园“第三空间”的关键。论文探讨了人本思想下读者对阅览空间的心理需求, 结合近年来阅览空间的发展趋势, 认为有必要在图书馆物 理空间内营造读者心理空间。通过引入格式塔心理学和视知觉的概念, 论述了如何运用相似性、封闭性、完形性、接近性等四个完形 法则来营造心理空间，在满足读者基本需要的同时兼顾了多层次、个性化的需求。

关键词 图书馆，阅览空间，心理空间，视知觉，格式塔心理学

1. 引言

随着新兴媒体和数字阅读的发展, 高校读者的阅读习 惯逐渐产生变化, 读者入馆人次和图书借阅量呈现逐年下 滑的态势。与此同时，在信息共享空间的引领下，图书馆 意识到除资源和服务外, “空间”对于读者的重要性，并以 旧馆改造和新馆建设的方式积极转型。这一过程中, 藏书 量和阅览座位的数量不再是追求的主要指标, 更多的是在 分析读者情感、心理和行为的基础上对图书馆空间布局、 层次、色彩、光线等软环境的营造, 改善读者的身心体验, 为读者营造除寝室、教室之外的“第三空间”, 即“空间作为 服务”。这一转型的主要特点是: (1)藏书空间保持不变或被 压缩, 读者空间放在了更加重要的地位; (2)空间秩序重新 调整, 注重不同层次读者的多样需求, 营造多义空间或复

燕山大学青年教师自主研究计划课题（编号: 13SKB017）
合空间; (3)读者作为空间环境的主角, 室内装饰和家具 摆放以契合读者心理或情感需求来布置。

良好的空间体验已成为图书馆的核心价值之一 [1], 读 者从使用者拓展为空间环境的参与者和享受者, 实体的建 筑空间被打造为契合读者心理期望的空间形态, 以更好的 激发读者的阅读欲望和使用体验。而探寻并按照读者的心 理期望进行空间设计, 利用各种建构元素的造型、色彩、 材质和光线在单一的大空间内营造多样的读者心理空间, 成为新馆建设或旧馆改造的关键因素。

\section{2. 相关概念}

2.1 视知觉和格式塔心理学

人类在认识周围环境时, 感官因素中超过 $80 \%$ 的信息 
内容来自于视觉 [2]。大脑会对进入眼睛的光线刺激做出反 应, 在“看”的同时调动过往的经验和记忆进行解读、对比 并形成认知, 进而引起情绪的变化和生理上的反应。当看 到的景象与大脑中的记忆或期望匹配时, 情绪表现出亲切、 轻松、尊严、安全、自由等正面、积极的反应; 反之则会 带来紧张、焦虑、失望、气馁等消极的反应。从心理学上 来说, 视知觉是一种将到达眼睛的可见光信息解释, 并利 用其来计划或行动的能力[3]。

格式塔心理学又称完形心理学, 其核心内容是“人们认 识事物是对其整体特征的把握, 并不是简单的部分特性之 和, 整体拥有其构成部分没有的性质”[2]。例如两个书架的 平行摆放可以构成一个隔离空间, 而这个空间并不属于任 何一个书架的属性。格式塔心理学理论包含四个重要的的 组织原则, 即完形法则: 相似性、封闭性、完形性、接近 性。相似性是指某些具有相似属性的元素容易被看作一个 整体; 封闭性是指即对于属性相同或类似、不完全封闭的 界面, 知觉会有延长边线使其封闭并组成整体的倾向; 完 形性是指排列组合容易被联想为最简单、直接、规则图形 的物体趋于组成整体; 接近性是指越互相接近或临近的物 体, 越容易被视知觉组合成一个整体。

\section{2 心理空间}

当建筑落成后, 墙体、地板、天花板等明确的界面会 形成视觉可见、物理的内部空间, 而空间内的元素, 比如 家具、灯具、网柱、书架、装饰色彩、盆栽以及柔性材料 等会从心理上组成一个整体, 并与其他元素形成某种分隔, 这种分隔使人们从心理上能够感知到某种虚拟空间的存 在, 即建筑的心理空间[2]。心理空间不像实体空间那样有 具体的边界和尺度, 而只是在视知觉的作用下人们内心感 受到的特定区域。

\section{3. 新时期的图书馆阅览空间}

\section{1 读者的空间需求}

\section{1 .1 舒适感}

舒适感是一种综合感受, 包括光线的照度符合实际需 要, 声音既不嘈杂又不极度安静, 温度和湿度适宜, 通风 良好没有异味, 室内色彩富有层次, 冷暖色调协调搭配, 还包括环境的整洁、有序、温馨等, 舒适的环境给人带来 轻松、愉悦、满足的感受, 使人产生积极向上的心态; 反 之则会带来烦躁、压抑、郁闪的感受, 读者会有一种逃离 环境的欲望。

\section{1 .2 领域感}

爱德华·霍尔认为每个人周围都有一层无形的气泡, 气
泡内属于私人领域, 当人互相接近时气泡受到挤压, 人们 就会有紧张感, 并认为自己的私人领域被侵犯[4]。传统的 阅览空间中, 整齐排列的书架和桌椅营造了严肃、秩序的 环境, 能感受到较好的学习气氛, 但从读者行为来看, 其 布局和形式无法满足个性化的需求。以四人阅览桌为例, 前两位坐下的同学往往选择对角线的位置, 如果还有其他 空闲的阅览桌, 再来的读者将不会再加入到已有 2 人的阅 览桌中, 而是寻找更宽松的座位, “普遍有桌边优于中央, 靠墙胜于走道的心理和做法” [5]。另外, 其他诸如背向其他 读者、面向开窗或墙壁、围绕柱子、有适当遮挡的阅览桌 等视线相对不受干扰的位置更容易营造个人领域感, 更受 读者欢迎。

\subsection{3 私密性}

私密性指个人在某种情况下, 要求所处环境具有隔绝 外界干扰的作用[6]。其不同于独处, 更类似于能主动控制 与他人的相互影响, 避免视线、声音、肢体的相互干扰, 与领域感相比, 私密性的主动权更大。对于渴望安静学习 的读者来说, 避免视线相对的席位, 如背对其他读者、面 向窗外、大厅、墙壁或带有隔断的书桌, 或环绕网柱、楼 梯下、以及建筑的不规则角落空间等具有一定围合感和容 积感的位置更受青睐, 更有安全感, 私密性更强。

\section{1 .4 安全感}

安全感是指读者在心理上对所处环境的确定、信任和 掌控。人进入视野宽广的空间内, 总会在视觉的指引下根 据周围的事物判别方向、确认位置, 找出行动的依据[7], 而在停留时喜欢尽量接近有依靠的物体, 因为人们倾向于 在空旷的环境中寻找支持物。比如在大厅、走廊等休闲区 域, 如果放置双面座椅, 读者更喜欢面对人群或交通区域 而不是背对, 而在阅览区域, 靠墙或尽端的座椅更受欢迎。

\section{1 .5 便于交流}

交流是现代社会人类获取知识的重要渠道, 图书馆的 公共属性更应该提供思想碰撞的交流空间。交流分为有目 的的、正式的交流和偶发的、随意的交谈, 其中第二种情 况在普通阅览空间中更为常见。长时间阅读后的短暂休息、 学习中引发的讨论、同学间的偶遇都会引发多人之间的交 流, 而在安静的大环境中, 结伴或者有讨论需求的读者会 尽量远离其他读者并选择利于面对面交流的座位[8]。

\section{2 阅览空间的变化特点}

\section{2 .1 复合空间}

复合空间是指建筑空间具有多属性和多层次的特征 [9], 也有学者称为多义空间、多阶空间。图书馆复合空间 并不是多种功能在同一空间的简单叠加或累积, 而是各部 
分互相重叠、渗透、交叉、融合, 由多种功能形成的一个 完整生态系统, 各功能区域边界模糊, 必要时功能可以互 相补充和替代, 空间功能的界定更多的取决于读者在其中 的行为。在比较典型的复合空间中, 布置有多种类型甚至 异型的书架、桌椅、沙发或者坐垫、方発。家具以适合不 同读者需要的方式灵活摆放, 读者可坐、可卧甚至席地而 坐; 提供安静学习区、电子阅览区、休闲阅读区、讨论区 或研讨室, 甚至提供简单的咖啡厅或有馆员参与的学习共 享空间; 绿植、盆栽、软隔断等装饰元素穿插其中, 空间 在整体上保持连续、流通、渗透、穿插但并不凌乱。

\subsection{2 灵活多变}

灵活多变指空间功能的动态可调整[5]。传统的阅览室 功能设计缺乏弹性, 对读者数量、个性化需求的可扩展性 差。而空间的动态性一方面表现在整体建筑的模数式设计, 可以在建筑的整个生命周期内根据图书馆的发展和读者需 要进行功能区域的调整; 另一方面是指其复合空间的属性 可以满足不同专业、不同层次读者的需求, 以及不同时间 段读者流量的需要。比如考研的学生更喜欢安静的学习, 低年级读者倾向于休闲阅读, 小组学习偏向于讨论空间, 做设计的同学更喜欢有电源的阅览桌以方便使用电脑; 上 课时段读者较少, 可自由选择喜欢的阅读区域, 晚自习高 峰期间非正式学习区域则可以兼顾所有读者的需要, 弥补 座位需求。

\subsection{3 大空间, 小区域}

在营造复合空间的同时, 大空间的限定被淡化, 小空 间的需求被揭示出来[10]。大空间意味着开阔的视野和良好 的学习氛围, 但整齐摆放的书架和阅览桌椅常给人以严肃、 刻板的感觉。因此, 利用空间构件相对围合, 在视知觉的 作用下在大空间中营造灵活多样的心理空间, 达到隔而不 分、空间穿插、相互联系和渗透的效果, 成为满足读者个 性化学习需要的新方法。

\section{2 .4 数字化}

随着电子资源在图书馆知识供给中发挥着越来越重要 的作用, 读者对电源和无线网络的需求大大增加, 对于可 携带笔记本电脑进入的阅览空间, 个人阅览面积也应大于 传统阅览面积。这种变化一方面表现在空间可以包容多种 媒体, 提供无线网络环境和电源等人机交互的环境; 另一 方面需要规划相对合理的空间布局, 区分静音区和非静音 区, 以避免多媒体发出的声音影响安静学习的读者。

\subsection{5 空间多样性}

相对于复合空间的功能融合, 空间多样性着眼于视觉 效果, 即通过非规则的家具摆放方式, 在大空间内营造多 向、交叉的视觉感受。空间多样性在不妨碍空间功能的前
提下更加注重空间与读者的互动, 比如用书架的简单围合 形成稍微不规则或改变行进方向的空间界面, 使读者随着 自身位置、视线的变化带来较动态的视觉体验, 多样的空 间序列和空间界面形态会给人一种“柳暗花明又一村”的感 觉, 并且空间的变异可以激发读者的神秘感和好奇心, 缓 解长时间阅读后的视觉疲劳。

\section{3 营造心理空间的必要性}

建筑师在设计图书馆时主要考虑的是整体布局和功能 分区, 对读者心理预期涉及较少。对内部空间中的书架、 桌椅、家具和装饰物等实体元素进行合理的排列组合, 可 以经由读者的视知觉进行重新组织, 让读者以个体本身的 心理去感受其所处的物理空间, 在大空间中营造丰富多样 的心理空间氛围, 在满足共性的前提, 契合不同读者的个 性化心理需要, 以诱导读者安静学习、休闲阅读、小组研 讨或者仅仅是坐下来休息片刻。通过视知觉对物理空间的 再组织, 物理空间就会反映出个体的视知觉感受, 进而促 使物理空间和心理空间的相互融合，而这种实体空间与读 者心理空间的契合程度直接决定阅览空间的舒适度以及读 者对阅览空间乃至图书馆的满意度, 因此按照读者的尺度 和心理需求对空间做进一步划分, 是体现图书馆空间人性 化的重要方式。

\section{4. 心理空间的营造}

\section{1 相似性原则的运用}

人的视知觉能够感知到视觉范围内的各种类似的属 性, 比如视觉元素的大小、形状、色彩和材质, 并使它们 产生一定的联系和区分。对于心理空间的形成, 可能是其 中的某一元素起作用, 也可以是多个元素同时起作用。

阅览室内过多并且缺乏组织的元素如书架、桌椅、沙 发、盆栽、灯具等会带来凌乱、无序的感觉, 而对其中一 种元素的重复、规则化的布置, 会使读者自觉的对这些元 素进行重新视觉相似性组织, 进而构成心理空间, 使无序 的元素形成有层次感的有序空间。比如整齐排列的书架和 阅览桌, 虽然两个区域之间并没有任何的阻隔, 但读者仍 能非常强烈的感受到陈列空间和学习空间两个不同的区 域。另外, 图书馆往往利用整齐排列的柱子所形成的视觉 张力和韵律感分割空间, 以柱体之间的连线为分割线, 划 分不同的功能区域。当网柱之间的距离越短时, 他们之间 的聚合力越大, 其分割作用越容易被读者感知。

装修中常用的格栅天花板, 虽然其在结构上没有连成 一块, 但紧密的相似性排列仍然能形成一个明显的心理界 面, 使读者感受到空间的高度而忽略上面的管道空间。过 于空旷的大厅或层高较高的空间内, 可以用下垂灯具的相 
似性排列, 在半空中形成心理界面以减缓其空旷感, 或者 长短不一的下垂线形成立体的坡度或者波浪感, 同样可以 在不改变空间尺度的情况下，使立体空间变得富有活力。

另外, 还可以利用打破相似性表达的方法, 对心理空 间进行分隔, 即元素的非相似性表达。在相似的元素中加 入对比明显的色彩、纹理, 利用元素明显的差异带来的分 隔界面, 使视知觉感知到两种异质元素发生转换的交界面 产生心理分隔, 进而形成不同的心理空间。比如整齐排列 的阅览桌椅之间加入一个与桌椅颜色形成对比色的沙发, 那么原有的独立空间很容易就被读者从心理上解读为沙发 两侧的相对独立的两个空间, 使空间变得更加丰富; 排列 整齐的书架中插入其他颜色或大小不同的书架, 则能够提 示读者从此处开始, 两侧书架所藏书刊的学科发生了变化。

冷暖或深浅对比色发生变化的位置、粗䊅和光滑界面 交接的位置、不同方向的条纹或者纹理交接的位置同样容 易产生心理界面, 形成心理空间[11]。比如图书馆的地板, 可以通过改变其铺设区域的颜色, 也可以利用不同的地板 材质区分不同的功能区域, 使之在不对空间整体性产生任 何影响的基础上, 让读者感知到心理上的分隔, 从而对空 间按照不同功能进行划分, 不同颜色的天花板同样能达到 这一效果, 而如果地板和天花板的颜色相互呼应, 则这种 心理分割更加明显。在大空间或者阅览区域的读者流线上 铺设有别于地板色彩的其他颜色地毯, 则能在地毯和地板 交接的地方形成立体的心理界面, 人为的为读者规划人行 通道, 解决读者在大空间穿行容易抄近路打扰其他读者的 问题, 并可以借此为读者导向, 起到平面导引的作用。

\section{2 封闭性原则的运用}

我们的知觉对不完满的图形有一种使其完满的倾向, 即填补缺口的趋向 [12], 知觉会在缺口处继续沿实体元素原 有的方向延长。比如把封闭房间四周的墙体全部拆掉, 仅 保留角落部分的墙体, 我们仍然明显的感受到四个角所围 合成的区域, 这就是封闭性原则在引导视知觉对视觉元素 进行组织而达到的效果。

除上述用色彩或纹路营造心理空间外, 适当抬高的地 板、稍微降低的天花板都会在视知觉的引导下, 以抬高或 降低的形状边缘为延长线, 形成向上或者向下的心理界面, 并形成有别于大平面的心理空间。其他诸如因建筑形体的 不规则所形成的角落空间, 也容易利用墙体的遮掩在封闭 性原则的引导下形成较为私密的心理空间。如在大空间的 平面上铺设地板相对抬升的休闲阅读区, 可在台阶处形成 向上的心理界面; 汕头大学图书馆逐渐下沉的多级阅览空 间, 在垂直方向上给读者营造了多个层次、相对独立的心 理空间; 在大于交通所需的走廊一侧设置简易隔断, 可打
造具有较强封闭感的独立空间，形成休闲阅读区域。

封闭性组织原则在图书馆家具摆放中的应用更加普 遍。比如带有中线隔断的阅览桌, 中线隔断形成明显向上 的心理界面, 与对面的读者分隔成两个心理空间; 相对围 合、波浪形或向内凹陷的阅览桌, 均较容易在近距离内产 生领域感或空间感, 从心理上形成相对独立的个人空间, 满足私密性的需要。图书馆较为常用的还有弧形书架的组 合, 视知觉会把两个以上的弧形书架组织为一个整体, 并 把其围合的区域, 独立出一个圆形或制圆形的心理空间, 一般用来形成工具书阅览区; 或利用圆弧形的向心沙发、 $\mathrm{L}$ 形相对摆放的沙发等围合成明显的交谈区域。

增加一个界面, 界面会在视知觉的作用下从心理上顺 着界面延伸, 形成心理界面, 进而在实体空间中形成心理 空间。因此, 在大的空间中利用建构元素, 如下垂的建筑 横梁或软隔断、镂空的屏风、家具、大型盆栽、装饰书架、 吧台式的休闲阅读桌等, 对空间和功能进行划分, 既可以 灵活调整, 又能使平淡的空间变的丰富而有趣, 形成一种 流动、贯通并且隔而不离的空间。

\section{3 完形性原则的运用}

知觉有一种把视觉元素看成最简洁、完好图形的倾向, 并与记忆中常见的简单图形做比较, 如方形、圆形、三角 形或对称形状, 使复杂和难以把握的图形简化、完整和稳 定。比较复杂和不规则的空间不容易被感知, 读者会感到 迷惑甚至需要较多的心理思考, 但太过于直观的空间却又 缺乏趣味性。因此, 稍有复杂但又不非常直观的空间形态 往往在引起好奇心和紧张感的同时, 容易随着知觉的明确 形成完好的心理空间, 进而在心理上感觉到舒适和平静。

对完好的空间适当进行抽象化变形可以实现上述思 想, 比如香港中文大学图书馆的 150 米长的曲线学习桌, 读者会在短距离内把弧线看作圆形的一部分。三个或四个 相对摆放的单人沙发会使读者直观的联想到三角形和正方 形, 形成相对的心理空间。 $\mathrm{S}$ 曲线、波浪线、菱形、椭圆形、 太极图等稍微复杂一些的阅览席位布置方式, 在稍带趣味 和变幻的同时不脱离想象空间, 较容易被读者所感知, 同 时与大空间结合紧密, 互相渗透但又有所区别, 兼顾了秩 序感和心理空间的营造, 可以取得较好的效果。

除较多的运用在座椅的摆放等平面效果上, 完形性原 则更多的运用在立体效果或俯瞰图上。因为建筑结构和外 部造型的原因, 阅览空间平面往往不太规则, 为了更好的 引导读者利用空间, 在异型或者不规则空间中, 布置室内 元素或划分功能分区时对平面进行相对规则的划分, 使其 被看成两种或多种简单形状的交叠, 可以使读者更好的利 用知觉把建筑空间分解为心理空间, 从而区分同一平面的 
不同功能区域, 帮助读者更好地认知空间, 并使得复杂繁 琐的空间变得更为简单清晰, 利于读者使用。

\section{4 接近性原则的运用}

彼此空间紧密接近的视觉各元素比相隔较远的部分组 合在一起的趋向更大 $[13]$ 。在建筑空间中, 尽管元素可能具 有不同的大小、造型、材质、色彩等属性, 但是人们总倾 向于把距离非常接近的物体看作整体。接近性原则在阅览 空间中主要用于对不同色彩、材质和形状的书架、阅览桌 椅、沙发等元素进行组合, 在保持空间完整性或不破坏排 列韵律的情况下, 利用色彩或形状的变异活跃空间形态, 使空间元素的属性在并不相似的情况下仍能被知觉组合成 一个整体，并形成心理空间。

其常用的表现手法是外向性表达: 即元素的内向组合 形成相对独立的心理空间, 多个心理空间的相互接近组成 更大的心理空间。阅览空间内, 双人书桌往往比四人桌更 受读者欢迎, 因为在接近性原则的组织下, 书桌之间距离 的增大使每个书桌形成独立的心理空间, 其个人领域感要 明显强于四人书桌, 而沿窗边成排布置的阅览桌所在的阅 读区域形成更大的心理空间。颜色鲜艳的座椅因为与阅览 桌的距离更近, 从而因为接近性形成一个整体, 并与其他 座椅和阅览桌的组合分隔开来, 而多组席位的排列形成电 子阅览空间, 与其他空间区别开来, 在大空间内形成层次 丰富的心理空间。

对于接近性原则, 距离是决定其组织原则的重要因素, 距离越近则联系越紧密。为了突出空间中的某一元素, 可 以使其他元素彼此更加接近, 或使其与其他元素拉开距离, 使该元素与其他元素分属在两个不同的心理空间。如把同 一学科藏书的书架整齐排列, 其内部的组合力使之形成一 个陈列空间, 不同学科陈列空间之间的距离增大或对角线 布置, 即可以人为勾勒主通道, 又增加了空间的层次感, 方便读者识别; 同样的方式也可以用在阅览席位的摆放上。 把阅览室内具有展览性、标志性、向导性、装饰意义的元 素与其他事物拉开距离, 也都可以达到吸引读者视线, 突 出其特质的目的。

\section{5. 结语}

图书馆空间环境设计的重心, 已从单纯的效率性的物 用功能逐渐转向调节与启迪读者情绪与心理需求为主的阶 段, 从某种程度上说, 心理的舒适感要比使用的便捷性更 能获得认同[14]。而心理空间这种与传统的开敞空间或封闭 空间完全不同的形态, 通过功能区域的交错覆盖和穿插交 替, 使空间贯通但又自成一隅, 视线通透而又相对分隔, 在不破坏平面的同时营造功能丰富且井然有序的空间环
境, 在满足读者基本需要的同时兼顾多层次、个性化的需 求, 调节读者的心理情绪, 满足读者心理需求, 给读者以 良好的使用体验和感官效果, 使图书馆的人文思想得到进 一步发展。

\section{参考文献(References)}

[1] J.-B. Lang. "Space experience_ —one of the core value of library," Journal of Academic Libraries, no.02, pp.42-48, 2013.

[2] Q. Wu. The Research on Psychological Space of Architecture Based on Visual Perception, Qinhuangdao, CN: Zhejiang University, 2012.

[3] X. Wang. "Design of artificial light environment visual perception theory based residential construction," Sichuan Building Science, no.03, pp.244-245, 2013.

[4] M.-L. Li. "Environmental psychology in the elevator and the exploration of space design," Journal of Lanzhou Jiaotong University, no.02, pp.123-127, 2013.

[5] Y. Guo. Study on the Use of Appropriateness of Reading Space in University Library, Chengdu, CN:Southwest Jiaotong University, 2010.

[6] J.-L. Fan. The Research of Psychological Space in Topological Architecture, Tianjin, CN: Tianjin University, 2012.

[7] M.-S. Lou. Conduct Psychological Space, Xi'an, CN: Xi'an University of Architecture and Technology, 2006.

[8] H. Liu. Study on Humanity Design of Public Sphere in Modern University and College Library, Xi'an, $\mathrm{CN}$ : $\mathrm{Xi}^{`}$ an University of Architecture and Technology, 2011.

[9] Y.-Y. Wang. Study on the Composite Space of University Libraries, Qingdao, CN: Qingdao Technological University, 2009.

[10] H.-T. Chen and L. Wu. "The discussion of characteristic on functional layout and space organization of library in university in the future," Shanxi Architecture, no.23, pp.17-18, 2007.

[11] X. Wang. The New Trend of the Design of Modern University Library, Dalian, CN: Dalian University of Technology, 2007.

[12] Q.-P. Luo. "Visual perception and spatial organization Environment (3rd)," New Architecture, no.04, pp.20-25, 1989.

[13] Q.-P. Luo. "Visual perception and spatial organization Environment (1st)," New Architecture, no.04, pp.32-37, 1988.

[14] T.-Z. Zhang, Y.-K. Zhang and D. Wang. "Space Organization of Architecture and the Psychological Schemata," Huazhong Architecture, no.03, pp.65-67, 2006. 